\title{
UTILIZACIÓN DEL BIO-DIESEL EN TRACTORES AGRÍCOLAS ZETOR
}

Adilio Portella Valverde'

\section{INTRODUCCIÓN}

Es bien conocido que el consumo anual de energía en el mundo se incrementa anualmente. Mientras que en 1970 fue de 161.19 mil millones de GJ, en 1992 fue de 355.21 mil millones de GJ. Gran parte de este consumo (en 1990 fue el $87.7 \%$ ) lo representan las fuentes fósiles de energía, los cuales causan efectos muy negativos para el medio ambiente; además, no son renovables y sus reservas disminuyen cada año.

La producción agropecuaria en el mundo, y principalmente en los paises desarrollados, es dependiente del consumo del petróleo diesel. En la República Eslovaca en 1993 fue de 8,803 TJ (49.58\% del consumo total de energía en la agricultura), lo cual trae además problemas relacionados con el precio y la importación del petróleo.

La disminución de fuentes fósiles de energía asi como la necesidad de disminuir su consumo con la finalidad de proteger el medio ambiente, permiten la posibilidad de utilizar fuentes alternativas de energía. En los últimos 5 años en Europa, principalmente en Austria, Alemania, Francia e Italia, se ha dado mucha atención a la utilización de aceites vegetales como combustible para motores diesel.

En la actualidad en Europa Central tiene mayor significación la utilización de la colza para la producción de aceite vegetal, gracias a grandes superficies de cultivo, rendimientos alcanzados, tecnologias desarrolladas y bajos costos de producción.

El aceite de colza se puede utilizar directamente como combustible pero en motores especiales. La mejor solución es su conversión en metil éster (bio-diesel), el cual tiene similares propiedades a las del petróleo diesel. La conversión se realiza mediante el proceso bioquímico de «esterificación», que consiste en la separación de los

1. PhD en Tecnología y Mecanización agricola. triglicéridos mediante la acción de la mezcla de metanol y catalizador (óxido de sodio) lo que permite la obtención de moléculas más pequeñas que, a su vez, disminuyen su viscosidad y mejora el proceso de combustión.

Además del bio-diesel, se obtiene de la colza los residuos que se utilizan como alimento para el ganado y la glicerina como materia prima para la industria farmacéutica, de cosméticos y de jabones. De 3 toneladas de granos de colza se obtienen 1 tonelada de bio-diesel, 1.9 toneladas de residuos y 0.1 toneladas de glicerina.

De acuerdo con los pronósticos, los motores diesel seguirán siendo utilizados en el futuro para la propulsión de diferentes vehículos como: tractores agricolas, camiones, diferentes máquinas de trabajo y en aplicaciones estacionarias.

Las pruebas de comparación en laboratorios y en el campo de motores diesel y tractores agricolas utilizando el bio-diesel, realizadas en diferentes paises europeos, principalmente en Australia, demuestran que el bio-diesel puede ser utilizado en sustitución del petróleo diesel prácticamente sin ninguna modificación de motores. En la actualidad, en Europa, han sido recomendadas por los fabricantes, para utilizar el bio-diesel,las siguientes marcas de tractores agricolas: Ford, John Deere, Deutz Fahr, Massey Fergusson, Mercedes Benz, Same, Case, Fend, Steyr y Fiatagri.

Los programas de producción y utilización del biodiesel en Eslovaquia, se encuentra todavia en etapa de desarrollo y al suministro de tecnología propia para su producción se dedican 2 empresas eslovacas: ZTS VVU Martin (Centro de Investigación y Desarrollo de Maquinaria Pesada) y MARTIN DAVOS TADING (empresa privada).

\section{OBJETIVOS}

Este trabajo se propuso: evaluar las influencias de la utilización del bio-diesel en los parámetros técnicos, energéticos, ecológicos y de 
explotación de los factores agrícolas Zetor UR II, sin nungúna modificación en sus motores y realizar una evaluación económica de su producción y utilización en condiciones actuales en la República Eslovaca.

\section{METODOLOGÍA}

Para alcanzar estos objetivos, se escogió una metodologia de comparación cualitativa y cuantitativa, utilizando el bio-diesel y el petróleo diesel, como combustible. Además se ha utilizado el método de interacción de problemas: técnico-ecológicoeconómico y los métodos técnicos de las normas Checoslovacas: CSN 300415 y CSN 470120.

Las pruebas de comparación se realizaron en 2 tractores agricolas: Zetor-12245 (con motor de 114 HP) y Zetor -162 45 (con motor de $154 \mathrm{HP}$ ), sin ninguna modificación en sus motores y utilizando biodiesel fabricado en la Cooperativa Agricola SalgovceEslovaquia.

\section{Las pruebas realizadas fueron:}

1. Pruebas de laboratorio en dinamómetro

2. Pruebas de tiro en asfalto

3. Pruebas en labores agricolas

4. Pruebas de largo periodo

\section{RESULTADOS DE LAS PRUEBAS}

\section{PRUEBAS DE LABORATORIO EN DINAMÓMETRO:}

Estas pruebas de comparación se realizaron con la finalidad de obtener las carateristicas técnicas de los motores de ambos tractores, utilizando el biodisel y el petróleo diesel. Tractor Z-122 45 después de 530 y 1184 motohoras de trabajo y el tractor $Z-162$ después de 905 y 1940 motohoras de trabajo. Las mediciones se efectuaron en el laboratorio de la Cátedra de Vehiculos y Plantas Técnicas de la Universidad de Agricultura de Nitra con los siguientes resultados:

CUADRO N• 01: Diferencias en los parámetros en la velocidad nominal de los motores de ambos tractores, utilizando el diesel y el petróleo diesel como $100 \%$.

\begin{tabular}{|l|r|}
\hline \multicolumn{1}{|c|}{ PARÁMETROS } & DIFERENCIA (\%) \\
\hline Potencia del motor & -3.80 a -7.24 \\
Consumo de combustible por hora & +6.99 a 9.30 \\
Consumo especifico de combustible & +11.94 a 15.75 \\
Densidad del humo & -50.85 a -65.52 \\
\hline
\end{tabular}

\section{PRUEBA DE TIRO}

Estas pruebas se realizaron en el tractor $Z$ 12245 con la finalidad de obtener sus caracteristicas de tracción, utilizando el bio-diesel y el petróleo diesel. Las mediciones se efectuaron sobre asfalto con la ayuda de un vehículo especial de medición TATRA. 815 y con los siguientes resultados:

CUADRO N ${ }^{\circ}$ 02: Diferencia (\%) en los parámetros del tractor Z12245 en la fuerza de tiro óptima, utiliándo el bio-diesel como $100 \%$.

\begin{tabular}{|l|r|r|r|}
\hline \multirow{2}{*}{ PARAMETROS } & \multicolumn{3}{|c|}{ CAMBIO DE VELOCIDAD } \\
\cline { 2 - 4 } & $\mathbf{1 - 2}-\mathbf{z}$ & $\mathbf{1 - 3}-\mathbf{z}$ & $\mathbf{1 - 4 - \mathbf { z }}$ \\
\hline Fuerza de tiro óptima & -0.93 & -4.18 & -13.08 \\
Potencia de tiro & +0.95 & -6.80 & -5.00 \\
Velocidad del tractor & +1.94 & -2.73 & +9.35 \\
$\begin{array}{l}\text { Consumo de combustible por } \\
\text { hora }\end{array}$ & +21.68 & +10.89 & +15.26 \\
$\begin{array}{l}\text { Consumo especifico de com- } \\
\text { bustible }\end{array}$ & +20.50 & +18.70 & +21.38 \\
\hline
\end{tabular}

\section{PRUEBA EN LABORES AGRICOLAS}

Estas pruebas se realizaron en la Cooperativa Agricola "Salgovce» con la finalidad de comparar los parámetros energéticos y la explotación del tractor Z-122 45, utilizando el bio-diesel y el petróleo diesel.

Las mediciones se efectuaron en diferentes labores agricolas con los siguientes resultados:

CUADRO $N^{\circ} 03$ : Diferencia (\%) de los parámetros del tractor $Z$. 122 45, utilizando bio-diesel y petróleo diesel como $100 \%$.

\begin{tabular}{|l|c|c|c|c|}
\hline \multirow{2}{*}{\begin{tabular}{c}
\multicolumn{1}{|c|}{$\begin{array}{c}\text { LABORES } \\
\text { AGRICOLAS }\end{array}$} \\
\cline { 2 - 5 }
\end{tabular}} & $\begin{array}{c}\text { Velocidad } \\
\text { del tractor }\end{array}$ & $\begin{array}{c}\text { Rendi- } \\
\text { miento }\end{array}$ & $\begin{array}{c}\text { Cons. de } \\
\text { Comb./hora }\end{array}$ & $\begin{array}{c}\text { Cons. de } \\
\text { Comb./há }\end{array}$ \\
\hline Arado del rastrojo & -1.85 & -1.09 & +10.21 & +11.45 \\
$\begin{array}{l}\text { Arado profundo } \\
\text { Siembra de alberjas } \\
\text { (guisantes) }\end{array}$ & -0.84 & -2.00 & +9.64 & +11.88 \\
$\begin{array}{l}\text { Siembra de alfalfa } \\
\text { Cosecha de la } \\
\text { remolacha azucarera }\end{array}$ & -0.77 & -2.19 & +9.15 & -11.44 \\
\hline
\end{tabular}

\section{PRUEBA DE LARGO PERIODO}

Estas pruebas se realizaron con ambos tractores durante 2 años utilizando el bio-diesel en sustitución del petróleo diesel. El tractor Z - 12245 , trabajó $1412 \mathrm{Mh}$. en la Cooperativa Agricola "Salgovce" y el tractor Z -162 $452803 \mathrm{Mh}$. en la Cooperativa Agricola "Preselany» con los siguientes resultados: 
CUADRO N 04: Parametros de 1 año de ambos tractores, utilizando solamente bio-diesel.

\begin{tabular}{|l|c|r|r|}
\hline \multicolumn{1}{|c|}{ DESCRIPCIÓN } & UNIDAD & Z-122 45 & Z-162 45 \\
\hline Horas de trabajo & (h) & 778 & 846 \\
utilización del tiempo de trabajo & & 0.59 & 0.89 \\
$\begin{array}{l}\text { Combustible consumido } \\
\begin{array}{l}\text { Consumo de combustible por } \\
\text { hora }\end{array}\end{array}$ & (L) & 10575 & 13849 \\
\hline
\end{tabular}

Además de los parámetros energéticos y de explotación, se han detectado las siguientes influencias negativas del bio-diesel en el trabajo de los tractores:

- La distribución del aceite lubricante ha sido entre el 2 al $15 \%$ y en algunas veces más del $15 \%$. Más baja disolución resulta cuando el tractor trabaja en labores pesadas (arado) y más alta disolución resulta cuando el tractor trabaja en labores livianos (transporte).

- La utilización del bio-diesel origina en las toberas de las valvulas de inyección, corrosión y más desgaste y con ello la disminución de su vida útil del 30 al $50 \%$.

- En las mangueras del sistema de combustible causa daños, disminuyendo su vida útil del 30 al $50 \%$

- En las mangueras del sistema de combustible causa daños, disminuyendo su vida útil a 2,000 motohoras.

- El encendido de los motores es hasta $-5^{\circ} \mathrm{C}$ igual que el petróleo diesel, hasta - $10^{\circ} \mathrm{C}$ se empeora y por debajo de $10^{\circ} \mathrm{C}$ es prácticamente imposible.

- Daños en la pintura del tractor, en lugares donde ha habido contacto con el bio-diesel.

\section{RESULTADOS DE LAS EVALUACIONES ECONÓMICAS}

Las evaluaciones económicas de la producción y utilización del bio-diesel en la República Eslovaca, se efectuó en base a datos reales obtenidos en la Cooperativa Agrícola "Salgovce» (fabricante del Bio-diesel) y de los resultados de las pruebas de campo.

CUADRO N 05: Costos de Producción de $1 \mathrm{~L}$ de bio-diesel.

\begin{tabular}{|l|c|c|}
\hline \multicolumn{1}{|c|}{ DESCRIPCIÓN } & UNIDAD & VALOR \\
\hline Costos de producción en 1 ciclo & (USD) & 1018 \\
Ingresos por productos secundarios & (USD) & 267 \\
Cantidad de bio-diesel producido & (L) & 950 \\
Costos de 1 L de bio-diesel & (USD/L) & 0.79 \\
\hline
\end{tabular}

CUADRO $N^{\circ}$ 06: Comparación de costos por hectárea del consumo de bio-diesel y del petróleo diesel del tractor Z - 12245.

\begin{tabular}{|l|c|c|c|c|}
\hline \multirow{2}{*}{$\begin{array}{c}\text { LABORES } \\
\text { AGRICOLAS }\end{array}$} & \multicolumn{2}{|c|}{ COSTOS (USD/há) } & \multicolumn{2}{c|}{ DIFERENCIA } \\
\cline { 2 - 5 } & $\begin{array}{c}\text { Petróleo } \\
\text { Diesel }\end{array}$ & Bio-diesel & (USD) & $(\%)$ \\
\hline Arado del rastrojo & 7.87 & 11.37 & 3.50 & +44.07 \\
Arado profundo & 12.33 & 17.83 & 5.50 & +44.61 \\
$\begin{array}{l}\text { Siembra de alberjas } \\
\text { (guisantes) }\end{array}$ & 1.39 & 2.00 & 0.61 & +43.85 \\
$\begin{array}{l}\text { Siembra de alfalfa } \\
\text { Remolacha azucarera }\end{array}$ & 1.73 & 2.45 & 0.72 & +41.61 \\
\hline
\end{tabular}

\section{CONCLUSIONES}

En base a la literatura estudiada y a los resultados de todo el trabajo, se ha llegado a las siguientes conclusiones:

\section{EN EL ASPECTO ECOLÓGICO}

El metil éster del aceite de colza (bio-diesel), es un combustible ecológicamente limpio, por cuanto tiene las siguientes ventajas en comparación con el petróleo diesel:

- No contamina el suelo, el agua ni las plantas en caso de fugas.

- Disminuye la contaminación de la atmósfera con bióxido de carbono (efecto del invernadero).

- Los gases de escape contienen sólo la mitad de aldehidos y de materias aromáticas.

- La densidad del humo es 50.85 a $65.52 \%$ menor (ver cuadro 1).

- Lo más importante, prácticamente no emiten óxido de azufre (SO), los cuales causan lluvias ácidas.

La desventaja es que los gases de escape del bio-diesel, contienen alrededor de 10\% más monóxido de carbono y de óxido de nitrógeno que los gases del petróleo diesel.

\section{EN EL ASPECTO TÉCNICO Y DE EXPLOTACIÓN}

El bio-diesel puede ser utilizado en lugar del petróleo diesel en los tractores Zetor UR II, prácticamente sin ninguna modificación en sus motores, excepto las mangueras del sistema de combustible, por cuanto las variaciones en los parámetros técnicos y de explotación son mínimos y pasan desapercibidos por los tractoristas (ver cuadro $1 ; 2$ y 3 ). 


\section{EN EL ASPECTO ECONÓMICO}

La utilización del bio-diesel en la República Eslovaca, es actualmente limitada, por cuanto sus costos de producción son en $37.06 \%$ más altos que el precio comercial del petróleo diesel, el cual es de USD 0.58, incluyendo los impuestos; y los costos por hectárea de su consumo son en 41.61 al $4.24 \%$ más altos que los costos por hectárea por petróleo diesel (ver cuadro 5 y 6 ).

\section{APLICACIONES}

En base a los resultados del trabajo, podemos recomendar la utilización del metil éster del aceite de colza como combustible para los tractores Zetor UR II, pero efectuando las siguientes acciones:

- Utilizar en el sistema de combustible, mangueras especiales que sean resistentes a los efectos negativos del bio-diesel.

- Cambiar las toberas de las válvulas de inyección cada 2,500 motohoras (2 veces en el plazo de validez de la garantía).

- Cambiar el aceite de lubricación, cada 150 motohoras.

- En lo posible, utilizar los tractores en labores pesadas.

- Controlar y mantener la hermeticidad del sistema de combustible para evitar fugas a la parte externa del tractor.

- En períodos de invierno utilizar aditivos especiales para mejorar el encendido del motor.

- Utilizar siempre bio-diesel, cuya calidad corresponda a las normas PN-7601 (ZTS Martín) o ONORMC

\section{REFERENCIAS BIBLIOGRÁFICAS}

$B E D Y, E .:$ Enrgia pre Slovensko-Alternative energie. Brastislava, UVSZOPK, 1992

BIENERGIE: Erste Osterreichsche Agrar-ragginerie Aschach, Forschungs Entmicklunss und Verwertungasgesellschaft. Wieselburg 1991.

CVENGROS. M : Strojno - Technologické riesenie liniek na vyrobu bionafty z repkového elleja. Martín. ZTS VVU, 1991.

FRAÑO, M. PORTELLA, A. : Overovanie motorov Zetor prevádzkovanych na metylester repkového oleja (vyskumná správa), Martín, ZTS VVU, 1993.

HANCAROVA, D. : Rostlinné oleja ako palivo pro sppalovaci motor, Praha, NVTIZ, 1992.

KOLEKTIV AUTOROV: Netradicnné energetické zdroje y polnoh., Nitra, VSP, 1991
1190 (Austria).

En lo que se refiere a la producción y utilización del biodiesel en las condiciones actuales de la República Eslovaca, podemos afirmar que no es rentable. Por ahora debe ser utilizado en zonas protegidas como los Parques y Reservas Nacionales $y$ en lugares de alta contaminación ambiental, y, naturalmente, bajo la subvención del Estado.

Para disminuir la diferencia entre el precio comercial del petróleo diesel y los costos de producción del bio-diesel, es necesario efectuar en los próximos años las siguientes acciones:

- Imponer un impuesto ecológico a los derivados del petróleo.

- Elevar el rendimiento de la colza a un nivel de 2.5 a 3 toneladas por hectárea.

- Elevar el contenido de aceite de colza a un nivel minimo de $40 \%$.

- Utilizar mejor los productos secundarios, por ejemplo, el uso de los residuos de los granos de colza para la preparación de concentrados como alimento de ganado.

- Construir, con financiamiento del Estado, 3 grandes refinerias (1 en cada Región) con un rendimiento anual de 10 mil toneladas de bio-diesel cada una y asociarlas a las ya existentes, çara que la producción anual en Eslovaquia llegue a $\mathbf{4 0}$ mil toneladas de bio-diesel, que representaria el $20 \%$ del consumo anual de petróleo diesel en la agrícultura.

KOZAK, J.- POKORNY, Z. : Bionafta, Mechanizace zemedelstvi, 6 , 1993.

LONG. E.: Fill up with rape oil, Farmers wekly, 18, 1990,s. 36.

PETRANSKY, I.- PORTELLA, A. : Systémy znizovania energetickej nárocnosti techniky a tecnhologie y polnohospodárstve (vyskumná správa). Nitra. KV a TZ VSP. 1994.

HERMANSKY, B. - STOLL, I.: Energie pro 21. stoleti. Praha, CVNT, 1992.

POKORNY.Z.- KOZAK, J.: Biopaliva a nová agrarni politika eurospskej unie. Mechanizace zemédélstvi. 3, 1994.

PROJEKT "EUROBIOSIESEL" : Praktische landtechnik, 51991 